\title{
An approach to tackle negative effects in project management
}

\author{
Ivan V. Nevolin*, and Oleg E. Khrustalev \\ CEMI RAS, Nakhimovsky pr-t, 117418 Moscow, Russia
}

\begin{abstract}
We introduce a model to support scenario analysis while managing costs of high-tech project. If the project results from joint efforts of firms with one per single stage the model suggests budget redistribution in case of funding shortage or exchange rate volatility. Given negative outer effects for the project the model requires the project to be completed and this constraint forces firms to diminish their profit or to make a loan if needed. The model proposed suggests human-machine (expert) interaction to build scenario for analysis but is simpler compared to method of successive concessions from computational point of view.
\end{abstract}

\section{Introduction}

Planning is a standard practice in high-tech projects development: circumstances arise along the time, and they require an appropriate reaction and control. A number of acting bodies, engineering difficulties and complexity of production technology require risk management techniques in the project implementation [1]. Obstacles in these techniques application associate with the number of contractors, competitors, nomenclature, production constraints, etc. - the dimension of the problem design and production grows rapidly when it comes to project planning [2], and the variables in question must be coordinated in an appropriate way. An increasing dimension of the problem entails both quantitative issues (an increase in the number of variables) and structural ones. The decision-maker is forced to deal with indicators of different nature: technical (transportation capabilities, weight of units and assemblies, etc.), financial (underlying budget), production (compatibility of components from different suppliers, equipment requirements and labor conditions) and physical (for example, size or solidity) features. A single expert or even a group of experts in one field is unlikely to cope with the task of project management - generally, solution requires knowledge from various disciplines, as well as an understanding of how variation in one factor affects the values of others. However, special techniques - mathematical models and software - are used to coordinate multidisciplinary efforts $[3,4]$.

Risk management with instrumental support suggests decisions following two paths. First, one could ground on previous experience involving probabilistic methods. Second, one could implement scenario analysis to assess the impact of specific events without sufficient number of observations.

* Corresponding author: i.nevolin@cemi.rssi.ru 
Probabilistic methods result in statistical distribution estimates of project costs and schedule given the random risks. The project analysis starts from the point estimate $[5,6]$ : statistical estimates map technical specifications into costs for every single stage of the project. For example, given the engine development costs one could build a statistical function that maps technical specification into R\&D expenditure. Project schedule ensures the planned result and describes the base scenario subjected to risk. The latter affect the cost of single stages both upward and downward. Experts assess statistical distributions of risks and their cross-correlations. Further, project variables as well as relations between contractors form the simulation model to estimate statistical variations in the baseline budget. As a result, the decision-maker receives for analysis the probability distributions of the total project budget and costs of single stages. This information supports additional funding allocation between project stages and contractors.

Scenario analysis allows the decision-maker to assess sensitivity of the project indicators to predetermined distortions and effects of different origin whether external or internal. To simulate the state of the environment - conditions external to the project - it is convenient to operate with the concept of "scenario". Scenario is a set of single events that changes the state of the environment, or - in other words - a function that brings the environment out of its normal state adopted in the project planning. Thus, a demand for reaction arises as a decisionmaker's response to the certain scenario.

Every event in any scenario has two characteristics: presence (or absence) and scale. Given this, it is possible to define the main events affecting the project and implement them in as software modules. Combining these events by adjusting the modules the decision-maker builds various scenarios. If one of the project participants gets better off under some scenario, an appropriate share of funding could be redistributed to ensure project completion. Details of this approach are outlined below.

\section{Cost management in scenario analysis}

Given a sufficient level of abstraction, we discuss a project management model with two key features. First, it supports interconnection of project stages and production processes providing the decision-maker with some kit of building blocks. Second, the model reflects the development stages, production and demand for resources, i.e. includes a cost analysis. An economic result of the model calculation is a project budget corresponding to the production plan. Following general description we focus on the principles of resources redistribution between the project participants while specific production processes only clarify the balance relationships between input and output variables.

Rather often is a task of maximizing result (not in terms of profit, but in terms of coverage area for communication facilities, computing power, drug efficiency, etc.) at minimum costs. In this formulation, budget optimization under the changing environment is possible in the following manner: 1) maximize the benefit while minimizing the cost of the project implementation, or 2) redistribute budget between participants so that the project is implemented, albeit with a reduced profit margin.

The first approach treats the entire life cycle of the project and arising in this context is a classic problem of multi-criteria optimization. To adjust funding of the stages one could apply the method of successive concessions, and human-machine decision support systems supporting this method are known [7]. So, the project implementation in economic terms sounds like a task of effect maximization at minimum costs. Output of the project belongs to a finite and discrete set of options to implement a product or service. The discreteness of a set is determined by two aspects. First, the characteristics of equipment (or substances) do not change continuously: each part or component is an independent product that has specific dimensions, weight, price, etc. Secondly, only some combinations of specific components 
and assemblies give a truly viable device, that is, there is a compatibility problem that needs to be solved. These combinations could be obtained using morphological synthesis [8] and are thus external to the model in question. Further, for analysis purposes the set of options could be reduced to the Pareto frontier - non-dominated project outputs in terms of revenue and budget. The Pareto frontier, therefore, consists of outputs with a minimum budget at fixed profit or those with a maximum profit at fixed costs.

The second approach of cost optimization - the budget redistribution between project participants - is based on the production processes revision at each stage of the project given the fixed layout of a specific product. Let the minimum resource requirements and inputoutput relations to be known for each stage. Then the decision-maker can vary the cost structure without falling below the threshold value - one that ensures the project output. An optimization begins with an initial budget estimate and follows the less painful - for the project implementation - cost shortage. The most obvious solution to the problem in this case is to assign financing at the threshold level for each of participants. However, the correct solution foresees the following. First, the financing of some participant could be at a level higher than the threshold, but with an insufficient rate of return. This may affect the participant's investment or scientific and technical policy of the. Secondly, in case of funding below the threshold the project has still to be implemented. For this, a participant may require some debt. And then a question arises that can be answered to only by looking at the general state of the participant: is the enterprise capable of paying debt on market conditions, and if not, whether to subsidize the interest rate or develop other supporting mechanism.

Often these are the projects that compete in the high-tech sector as, for example, in the aerospace and defense industry - not the individual enterprises. Leaving the problems of coalitions formation outside the framework, as well as the solution of technical problems associated with the search for the technology layout, we describe a model for drawing up an optimal project budget structure. At the same time, the issues of technological and production links are exogenous to the model, and data about them are provided for calculations as ready to use. Also, issues of options specification for the project are outside the framework and their cost structures are considered to be known. Under this setting, the model makes it possible to answer the question: how to change the base budget of the entire project when the external conditions change for the entire industry or for an individual participating enterprise.

\section{Model formalisation}

After the general characteristics of the model and its capabilities consider a formal description. Let $K$ firms to implement a project jointly - index them with $k=1,2,3, K$. The whole project is divided into $K$ stages, and each firm develops only one of them. Firms use standard accounting forms, and we consider one that describes the cost structure of a stage. If the structure contains $n$ fields, then the budget of firm $k$ at time $t$ at discrete time is expressed as $f_{k}^{t}=\sum_{i=1}^{i=n} x_{i}^{t}[k]$, where $x_{i}^{t}[k]$ - the value of field $i$ for firm $k$ at time $t$. If a period $T$ is required to complete the project successfully, then the total project costs are $\sum_{t=1}^{T} \sum_{k=1}^{K} f_{k}^{t}$. Each firm requires a certain threshold funding annually so that nothing threatens the implementation of the project. In the model, this funding is calculated as follows. Let the production matrix $M^{t}(k)=\left[m_{i, j}^{t}(k)\right]_{i, j=1}^{n}$ of the firm $k$ be known with every element showing how many resources from field $i$ are required to use resource $j$ when implementing production plan of firm $k$ at time $t$. Matrix elements that correspond to zero costs, i.e. the fields of the standard form of cost structure with no expenditure on the stage, are also considered equal to zero. If $a_{i}^{t}(k)$ is the minimum cost at field $i$ for firm $k$ at time $t$, then $x_{i}^{t}[k] \geq a_{i}^{t}(k)$ and $x_{j}^{t}[k]=m_{i, j}^{t}(k) x_{i}^{t}[k] \geq a_{j}^{t}(k)$. Next, define the field of the cost structure reaching its constraint in budget variation. Formally, let the item $i_{0}$ to reach its 
threshold while the others lie in interior of feasible region. Then, non-zero costs are $x_{i_{0}}^{t}[k]=$ $a_{i_{0}}^{t}(k)$ and $x_{j}^{t}[k]=m_{i_{0}, j}^{t}(k) a_{i_{0}}^{t}[k] \geq a_{j}^{t}(k)$. Consequently, $a_{i_{0}}^{t}(k) \geq a_{j}^{t}(k) / m_{i_{0}, j}^{t}(k), j=$ $\overline{1, n}, k=\overline{1, K}, t=\overline{1, T}$. Given this the budget of firm $k$ at time $t$ cannot be lower than $\bar{f}_{k}^{t}=$ $\sum_{i=1}^{i=n} x_{i}^{t}[k]=\sum_{i=1}^{i=n} m_{i_{0}, j}^{t}(k) x_{i_{0}}^{t}[k]=a_{i_{0}}^{t}(k) \sum_{i=1}^{i=n} m_{i_{0}, j}^{t}(k)$.

This approach to threshold calculation has the following advantages. First, it is beneficiary in cases of predefined lower values of cost items such as labor, amortization of reserve or temporarily unloaded production facilities, etc. Secondly, from a computational point of view, it is more convenient to determine an item $i_{0}$ reaching its threshold. By definition, $m_{i, j}^{t}(k)=x_{j}^{t}[k] / x_{i}^{t}[k]$ for nonzero elements, therefore resulting in $m_{i, i}^{t}(k)=$ $x_{i}^{t}[k] / x_{i}^{t}[k]=1$ and $a_{i_{0}}^{t}(k)=\max _{i, j}\left[a_{j}^{t}(k) / m_{i, j}^{t}(k)\right]$.

After the funding threshold calculation, we proceed to budget distribution at time $t$ between the firms involved. Consider an enterprise $k$ which receiving funding of $f_{k}^{t}$ at time $t$. From a computational point of view, it is convenient to operate with $\left(f_{k}^{t}-\bar{f}_{k}^{t}\right)$-the excess (or deficient in case of negative value) financing compared to base one ensuring the project implementation. We distribute the difference between costs items in appropriate proportions and the firm's profit in case of excess funding or demand for a debt otherwise. In case of the positive outcome a cost item $x_{j}^{t}[k]$ of firm $k$ increases proportionally taking form of:

$$
x_{j}^{t}[k]=m_{i_{0}, j}^{t}(k)\left[a_{i_{0}}^{t}(k)+\frac{f_{k}^{t}-\bar{f}_{k}^{t}\left(1+\pi_{k}^{t}\right)}{\left(1+\pi_{k}^{t}\right) \sum_{i=1}^{i=n} m_{i_{0}, j}^{t}(k)}\right]
$$

where $\pi_{k}^{t}$ is a rate of return for firm $k$ at time $t$.

If the outcome of $\left(f_{k}^{t}-\bar{f}_{k}^{t}\right)$ difference is negative, the cost item takes a threshold value while the firm makes a debt of $\mathrm{c}=\left(f_{k}^{t}-\bar{f}_{k}^{t}\right)$ for $\tau$ years with an annual rate of $r_{k}^{t}$. Therefore, cost items take a form of:

$$
\begin{gathered}
x_{j}^{t}[k]=m_{i_{0}, j}^{t}(k) a_{i_{0}}^{t}(k) \\
x_{n}^{t+i, t}[k]=\frac{c}{\tau}+\frac{c(\tau-i)}{\tau} r_{k}^{t}, i \in \mathbb{N}
\end{gathered}
$$

where $x_{n}^{t+i, t}[k]$ states for increased costs at time $(t+i)$ due to the debt at time $t$. With no loss in generality let this cost item be indexed with $n$. Note, in the above case of $\left(f_{k}^{t}-\bar{f}_{k}^{t}\right) \geq$ 0 no debt at time $t$ is made and thus $x_{n}^{t+i, t}[k] \equiv 0, i \in \mathbb{N}$. The potential payments because of debt entail a revision of the firm's budget. Formally, the costs of the firm under the item $n$ take a form of:

$$
\tilde{x}_{n}^{t}[k]=x_{n}^{t}[k]+\sum_{i=0}^{t-1} x_{n}^{t, 1}[k]
$$

Substantially, the following happens. Budget financing of the firm $k$ at time $t$ under some scenario is expected to be $f_{k}^{t}$. If it turns out to be less than the threshold value of $\bar{f}_{k}^{t}$, the firm makes a debt. In fact, a sum of the cost items results in firm's budget $\bar{f}_{k}^{t}$ for the project with insufficient funding being complemented by loans. But the firm pays for complete budget at time $t$ with increased expenses due to interests in next periods. In case of directive rates of return this means an increase in the total project costs - whether prom public sources or commercial ones - by the amount of credit payments. This growth demonstrates the decisionmaker that participation in the project becomes much less profitable compared to the baseline scenario as a debt is needed. And the amount of credit payments denotes withdrawals arise from income that other projects participants have to face in case of joint efforts. These withdrawals though could also be offset from the public sources with the project budget 
adjustment. The letter - increased project developments costs - is rather general in aerospace and defence [9]. Therefore, we arm a decision-maker with a tool supporting budget estimation in case of financing shortage for a single project stage in some time period.

The model suggests the project budget adjustments not only in case of reduced funding, but also under exchange rate fluctuations. Accounting for the budget dependence on imported components requires some modification in the above formulas.

Let the base budget $f_{k}^{t}$ of firm $k$ at time $t$ ground on the exchange rate $p_{0}^{t}$, the share of imported components $d_{k}^{t}$ in the cost of raw materials $x_{1}^{t}[k]$, without loss of generality, indexed with one. Then the change in the currency rate to $p_{1}^{t}$ increases the cost on

$$
x_{1}^{t}[k] \frac{p_{1}^{t}-p_{0}^{t}}{p_{0}^{t}} d_{k}^{t}
$$

Therefore, a scenario with exchange rate fluctuations the above schema to calculate cost items entails replacements in formulas:

1) of cost items

$$
x_{1}^{t}[k] \rightarrow x_{1}^{t}[k]\left\{1+\frac{p_{1}^{t}-p_{0}^{t}}{p_{0}^{t}} d_{k}^{t}\right\}
$$

2) of constraints

$$
a_{1}^{t}(k) \rightarrow a_{1}^{t}(k)+x_{1}^{t}[k] \frac{p_{1}^{t}-p_{0}^{t}}{p_{0}^{t}} d_{k}^{t}
$$

With calculations remain the same under replacements the model suggests a tool of processing a superposition of events forming scenarios. If a single event leaves the rate to be stable, neither demand for marginal costs nor constraints require modifications. If there is a for debt in a certain period, the cost of the debt becomes an extra funding to the general project budget.

\section{Conclusion}

The model described processes a set of parameters and variables. The first are budget of the project, the distribution of financing among the stages, production links between the stages, interest rates, resource restrictions, the share of imported components and the exchange rate. An analyst quantifies their values and depending on the settings, the cost structure is calculated as a vector of variables. Feeding the model with a certain set of input values, the analyst initializes a specific scenario, and as a result, she receives the best budget distribution for this particular scenario. Variation in at least one of parameters creates a new scenario. The analysis and selection of a specific financing schema, therefore, relies on the results of the scenarios calculation. Competition in the model doesn't take a form of participants' aspiration for larger share of budget, but looks like alternative solutions contest, which results in the project budget changes, resources redistribution between stages, choice of production processes and financial sources. The formalization of the model imposes requirements on the data: production costs, schedule and description of processes become critical.

\section{Acknowledgment}

The article was prepared with financial support by the Russian Science Foundation, grant № 21-78-20001 «Development of the theory and model tools for optimizing the management of 
the diversification of defense production in the context of the economic crisis and growing threats to Russia's national security»

\section{References}

1. NASA, NASA cost estimating handbook (2008)

2. D. Motte, R. Bjärnemo, In Proceedings of the 25th International Conference on Design Theory and Methodology - DETC/DTM'13, 5, (2013)

3. M. Mukelabai, D. Nešić, S. Maro, T. Berger, J.P. Steghöfer, In Proceedings of the 33rd ACM/IEEE International Conference on Automated Software Engineering (2018)

4. A.M. Batkovskiy, E.G. Semenova, A.V. Fomina, E. Iu. Khrustalev, O.E. Khrustalev, Indian Journal of Science and Technology, 9, 20 (2016)

5. G.S. Levenson, H.E. Boren, D.P. Tihansky, F. Timson, Cost-estimating relationships for aircraft airframes (1972)

6. F.D. Harris, M.P. Scully, Journal of the American Helicopter Society 43, 1 (1998)

7. Golshein E.G., Borisova E.P., Dubson M.S. Economics and mathematical methods, 26, 4 (1990)

8. A.V. Andreichikov, O.N. Andreichikova, Journal of Computer and Systems Sciences International 45, 4 (2006)

9. M.V. Arena, R.S. Leonard, S.E. Murray, O. Younossi, Historical Cost Growth of Completed Weapon System Programs (2006) 